\title{
SOME FIXED POINT PROPERTY FOR MULTIVALUED NONEXPANSIVE MAPPINGS IN BANACH SPACES
}

\author{
ZHANFEI ZUO
}

Abstract. We show some geometric conditions on a Banach space $X$ concerning the generalized James constant, the generalized Jordan-von Neumann constant, the generalized Zbagănu constant and the coefficient $R(1, X)$, which imply the existence of fixed points for multivalued nonexpansive mappings. Our results extend and improve some recent results.

Mathematics subject classification (2010): Primary 47H10, Secondary 46B20.

Keywords and phrases: Multivalued nonexpansive mapping, fixed point, generalized James constant, generalized Jordan-von Neumann constant, generalized Zbagănu constant.

\section{REFERENCES}

[1] S. B. NAdler JR., Multivalued contraction mappings, Pacific J. Math. 30 (1969), 475-488.

[2] W. A. KIRK, A fixed point theorem for mappings which do not increase distances, Amer. Math. Monthly 72 (1965), 1004-1006.

[3] S. Dhompongsa, A. Kaewcharoen, A. Kaewkhao, The Domínguez-Lorenzo condition and multivalued nonexpansive mappings, Nonlinear Anal. 64 (2006), 958-970.

[4] S. Dhompongsa, T. Domínguez Benavides, A. Kaewcharoen, A. Kaewkhao, B. PaNYANAK, The Jordan-von Neumann constant and fixed points for multivalued nonexpansive mappings, J. Math. Anal. Appl. 320 (2006), 916-927.

[5] A. KAEW KHAO, The James constant, the Jordan-von Neumann constant weak orthogonality and fixed points for multivalued mappings, Journal of Mathematical Analysis and Applications 333, 2 (2007), 950-958.

[6] B. GAVIRA, Some geometric conditions which imply the fixed point property for multivalued nonexpansive mappings, Journal of Mathematical Analysis and Applications 339, 1 (2008), 680-690.

[7] T. DomíngueZ BEnAVIDES AND B. GAVIRA, The fixed point property for multivalued nonexpansive mappings, Journal of Mathematical Analysis and Applications 328, 2 (2007), 1471-1483.

[8] T. Domínguez Benavides And B. Gavira, Does Kirk's theorem hold for multivalued nonexpansive mappings?, Fixed Point Theory and Applications Vol. 2010 (2010), Article ID 546761, 20 pages.

[9] J. GAO AND K.-S. LAU, On two classes of Banach spaces with uniform normal structure, Studia Math. 99, 1 (1991), 41-56.

[10] A. Jiménez-Melado, E. Llorens-Fuster, And S. Saejung, The von Neumann-Jordan constant weak orthogonality and normal structure in Banach spaces, Proc. Amer. Math. Soc. 134 (2006), 355-364.

[11] S. SAEJUnG, On James and von Neumann-Jordan constants and sufficient conditions for the fixed point property, J. Math. Anal. Appl. 323 (2006), 1018-1024.

[12] ZHANFEI ZUO, YUNAN CUI, On some parameters and the fixed point property for multivalued nonexpansive mapping, Journal of Mathematical Science: Advances and Applications 1 (2008), 183-199.

[13] Zhanfei Zuo, Yunan Cui, A note on the modulus of $U$-convexity and modulus of $W^{*}$-convexity, Journal of Inequalities in Pure and Applied Mathematics 9, 4 (2008), 1-7.

[14] Zhanfei Zuo, Yunan CuI, Some modulus and normal structure in Banach space, Journal of Inequalities and Applications Vol. 2009 (2009), Article ID 676373.

[15] Zhanfei ZuO, YUNAN CUI, A coefficient related to some geometrical properties of Banach space, Journal of Inequalities and Applications, Vol. 2009 (2009), Article ID 934321. 
[16] Zhanfei Zuo, Yunan Cui, The application of generalization modulus of convexity in fixed point theory, Journal of Natural Science of Heilongjiang University 2 (2009), 206-210.

[17] Zhanfei Zuo, YunAN CUI, Some sufficient conditions for fixed points of multivalued nonexpansive mappings, Fixed Point Theory and Applications, Vol. 2009 (2009), Article ID 319804.

[18] J. A. Clarkson, Uniformly convex spaces, Transactions of the American Mathematical Society 40, 3 (1936), 396-414.

[19] B. Sims, Orthogonality and fixed points of nonexpansive maps, Proc. Centre Math. Anal. Austral. Nat. Univ., Canberra 20 (1988), 178-186.

[20] T. Domínguez Benavides, A geometrical coefficient implying the fixed point property and stability results, Houston Journal of Mathematics 22 (1996), 835-849.

[21] E. M. MAZCUÑáN-NAVARRo, Banach spaces properties sufficient for normal structure, J. Math. Anal. Appl. 337 (2008), 197-218. 